\title{
Educación continua y fortalecimiento del desempeño del rol comunitario de enfermeros serumistas
}

Continuing education and strengthening the performance of the community role of serumista nurses

Mely Ruiz-Aquino ${ }^{1}$

María Luz Ortiz Aguí 2

Wilmer Espinoza Torres ${ }^{3}$

Gianny R. Garay Cabrera ${ }^{4}$

Elsa Elvira Palacios Flores 5

RECIBIDO: 4 DE MARZO DE 2020

ACEPTADO: 11 DE JUNIO DE 2020

\section{RESUMEN}

Objetivo. Demostrar la efectividad de la educación continua en el fortalecimiento del desempeño del rol comunitario de enfermeros serumistas del Consejo Regional XII de Huánuco. Metodología. Fue una investigación de tipo experimental-

\footnotetext{
1 Magister en Salud Pública y Gestión Sanitaria, Licenciada en Enfermería, Docente, Universidad de Huánuco, Huánuco, Perú; melyruizaquino@udh.edu.pe, ORCID: https://orcid.org/0000-0002-8340-7898 Google Scholar: https://scholar.google.es/citations?hl=es\&user=dw_z9NgAAAAJ

2 Doctora en Enfermería, Magister en Epidemiologia, Licenciada en Enfermería, Universidad Nacional Hermilio Valdizán, Huánuco, Perú; maorcru@hotmail.com, ORCID: https:// https://orcid.org/0000-0002-5613-7378 Google Scholar: https://scholar.google.com/citations?hl=es\&user=peljvfQAAAAJ

3 Licenciado en Enfermería, Universidad de Huánuco, Huánuco, Perú; wilmer.espinoza@udh.edu.pe, ORCID: https://orcid.org/0000-0002-9104-7722

Google Scholar: https://scholar.google.es/citations?hl=es\&user=GXf3hrQAAAAJ

${ }^{4}$ Magister en Salud Pública y Gestión Sanitaria, Licenciada en Enfermería, Hospital Materno Infantil Carlos Showing Ferrari, Huánuco, Perú. gianny_gc@hotmail.com, ORCID: https://orcid.org/0000-0002-2003-9346 Google Scholar: https://scholar.google.es/citations?hl=es\&user=521_dNcAAAAJ

5 Magister en Salud Pública, Licenciada en Enfermería, Universidad Nacional Hermilio Valdizán, Huánuco, Perú. epalacios@gmail.com, ORCID: http://orcid.org/0000-0002-8040-3306

Google Scholar: https://scholar.google.es/citations?hl=es\&user=GXf3hrQAAAAJ
}

Socialium, revista científica de Ciencias Sociales, Vol 4 - No. 2, julio - diciembre 2020, pág. 29-46. 
longitudinal, con dos grupos (experimental y control), conformados por 58 enfermeros en cada grupo, seleccionados por muestreo probabilístico aleatorio. Previo al programa de educación continua, se aplicó un cuestionario de desempeño del rol comunitario previamente validado. Se tuvo en cuenta las consideraciones éticas. En el análisis inferencial se aplicó la prueba t de Student. Resultados. Se observó que antes de la intervención, solo el 1,7 \% (1) del grupo experimental tuvo desempeño del rol comunitario de nivel alto, 89, 7 \% (52) de buen nivel y 8,6\% (5) de nivel deficiente; por otro lado, 98, 3 \% (57) del grupo control tenían buen nivel y $1,7 \%$ (1) de nivel deficiente. Después de la intervención, el 86,2 \% (50) del grupo experimental evidenció un alto nivel y el 13,8\% (8) un buen nivel. Solo el 1,7\% (1) del grupo control alcanzó un alto nivel y el 98,3 \% (57) un buen nivel. Se comprobó la existencia de diferencias significativas entre los promedios de los momentos y grupos de estudio $[t=-19,301 ; p=0,000]$, siendo mayor en el grupo experimental, por lo se admite que la educación continua fortalece el desempeño del rol comunitario de los enfermeros serumistas del grupo experimental. Conclusión. La educación continua fortalece el desempeño de rol comunitario de los enfermeros serumistas, habiéndose evidenciado diferencias significativas en los promedios entre grupos y durante el postest $[t=15,601 ; p=0,000]$.

Palabras clave: educación continua, rol comunitario, desempeño laboral enfermería, promoción de la salud, atención primaria de la salud

\section{ABSTRACT}

Objective. To demonstrate the effectiveness of continuing education in strengthening the performance of the community role of serumista nurses of Regional Council XII in Huánuco. Methodology. It was an experimental-longitudinal research, with two groups (experimental and control), composed of 58 nurses in each group, selected by random probability sampling. Prior to the continuing education program, a previously validated community role performance questionnaire was applied. Ethical considerations were taken into account. Student's t-test was applied for inferential analysis. Results. It was observed that before the intervention, only $1.7 \%$ (1) of the experimental group had high-level community role performance, $89.7 \%$ (52) of good level and $8.6 \%$ (5) of poor level. On the other hand, $98.3 \%$ (57) of the control group 
had a good level and $1.7 \%$ (1) had a poor level. After the intervention, $86.2 \%$ (50) of the experimental group showed a high level and $13.8 \%$ (8) a good level. Only $1.7 \%$ (1) of the control group reached a high level and $98.3 \%$ (57) a good level. The existence of significant differences between the averages of the moments and study groups was verified $[t=-19.301 ; p=0.000]$, being higher in the experimental group, therefore it is admitted that continuing education strengthens the performance of the community role of serumista nurses in the experimental group. Conclusion. Continuing education strengthens the community role performance of serumista nurses, showing significant differences in the averages between groups and during the post-test [ $\dagger=$ $15.601 ; p=0.000]$.

Keywords: continuing education, community role, nursing job performance, health promotion, primary health care

\section{Introducción}

Los nuevos retos que viene implementando el Ministerio de Salud (MINSA), requieren desarrollar y fortalecer las competencias de su recurso humano, a fin de mejorar su desempeño laboral; de modo que es necesario alinear este con los objetivos y estrategias del sector (Dirección General de Gestión del Desarrollo de Recursos Humanos del Minsa 2017).

El Servicio Rural y Urbano Marginal de Salud (SERUMS), obligatorio para todos los profesionales de las ciencias de la salud, titulados y colegiados, se ha convertido en la estrategia pública que el Estado peruano ha diseñado para cubrir la atención primaria de la salud (APS), a la población más vulnerable que reside en las zonas de menor desarrollo del país (Castillon, 2015); como, por ejemplo, el binomio madre e hijo/a (Castillon, 2015).

En este sentido, el profesional de enfermería, como reconoce Castillon (2015), debe ser continuamente capacitado, para hacer frente a los innumerables retos existentes en los establecimientos de salud; de modo que este se encuentre comprometido con el cuidado de las personas, las familias o las comunidades en las que trabaja (Zarate, 2004). Además, en el actual mundo globalizado, dichos profesionales 
afrontan la tarea de perfeccionar sus procesos de trabajo con vistas a garantizar los cuidados con calidad (Willamowius y Misue, 2009).

Por lo tanto, la capacitación del potencial humano se presenta como uno de los pilares esenciales que contribuyen con el desarrollo y el proceso de transformación hacia sistemas de salud basados en la APS (Castillon, 2015) con el fin de guiar los cambios en el sector salud hacia servicios accesibles, de calidad y merecedores de la confianza de toda la ciudadanía. La importancia de fortalecer las competencias en el rol comunitario a los nuevos profesionales de enfermería de manera estructurada y estandarizada es, según Nebot Adell et al. (2009), imprescindible para la consecución de los objetivos, mejorar la calidad de la atención, del cuidado y, por lo tanto, contribuir con la satisfacción del usuario externo; consecuentemente mejorar el desempeño laboral.

En este marco, los profesionales SERUMS (serumistas) de la carrera de enfermería, deben conocer, coordinar, dirigir y evaluar los programas y estrategias para el cuidado comunitario de la población donde desempeñan su trabajo (Gómez y Llontop, 2014). Sus funciones, reconocidas como esenciales en el Sistema Nacional de Salud, colocan a los servicios sanitarios al alcance del individuo, la familia y la comunidad; de este modo satisfacen las demandas en salud a lo largo del ciclo vital (Torres, Dandicourt y Rodríguez, 2005).

Resulta no menos importante tratar de minimizar, no obstante, el "choque cultural" que estos profesionales recién egresados enfrentan, durante el inicio de su desempeño laboral en los establecimientos de salud periféricos. Las poblaciones a las que interviene poseen en muchos casos patrones culturales diferentes al suyo. Por lo que, siguiendo la experiencia de Saavedra (2014), resulta difícil ofrecer una cobertura adecuada a las directivas emanadas verticalmente desde el MINSA, en aquellas comunidades distantes y de difícil acceso geográfico, económico y cultural. En muchos casos el profesional de enfermería no logra posicionarse allí como agente de cambio en salud; siendo otros profesionales de la salud quienes consiguen ser reconocidos por la comunidad como tales (Saavedra, 2014).

En consonancia con ello, el Colegio de Enfermeros del Perú ha dispuesto a nivel Nacional, a través de los consejos regionales y previo al otorgamiento de la colegiatura, que se fortalezca el desempeño de los profesionales; por lo que el 
Consejo Regional XII (CR-12) de Huánuco se encuentra implementando programas de educación continua entre los enfermeros/as, con énfasis en el rol comunitario. Este rol, tal como expresan Torres Esperón et al. (2005), refleja además las transformaciones que ha sufrido el concepto de salud en el transcurso de los años; por lo que coinciden con Feliu (1998), quien expresa que los profesionales de la enfermería realizan un aporte esencial a la salud de la población en el contexto actual, desempeñándose como promotores del desarrollo humano, en tanto que no solo proporcionan cuidados de salud, sino que además fomentan valores orientados hacia el mejoramiento de la salud de las personas, las familias y las comunidades (Ortiz et al. 2019).

El programa de formación continua que se desarrolló, pretende ser una herramienta de fortalecimiento del rol comunitario de los profesionales de la enfermería en el marco de la APS; lo que permitirá alcanzar cambios reales en la estructura y dinámica de dichos centros (Ministerio de Salud, 1999), brindando atención de calidad y sostenibles a aquellos usuarios a lo largo de su ciclo vital, tanto con intervenciones individuales como grupales.

El enfermero comunitario tiene la misión de resolver desde la APS, los problemas relacionados con la necesidad de cuidados; siendo estos especialmente importantes en determinados grupos de población, como niños, adolescentes, personas con enfermedades crónicas y personas mayores. Para la atención de la población objetivo, aplica protocolos y guías de práctica clínica que promociona la salud, mejoran los problemas de salud y minimiza las complicaciones; contando también con intervenciones en la resolución de problemas específicos y la aplicación de barreras frente a diversos factores de riesgo. Todo ello, en articulación con los demás profesionales de la salud. De modo que resultará positivo en la satisfacción de los usuarios y aumentará la eficiencia del sistema sanitario (Federación de Asociaciones de Enfermería Comunitaria y Atención Primaria, 2001).

El enfermero comunitario es, por lo tanto, un profesional que asume, a través de enfoque integral y holista, la misión de acompañar a las personas de manera personalizada (Ramos y Robles, 2016). Por lo que requiere de habilidades cognitivas, una preparación académica y aptitudes científicas, técnicas y humanas para 
orientar a cada individuo en su entorno (familiar, laboral y social), hacia el desarrollo de su potencial de salud.

Por consiguiente, se propuso esta investigación que aborda la educación continua y el fortalecimiento del desempeño del rol comunitario de los enfermeros serumistas inscritos en el Consejo Regional XII de Huánuco, durante el 2017; a partir de cuyos hallazgos se busca propiciar el empoderamiento profesional del proceso de renovación hacia sistemas de salud basados en la APS, enfatizando la promoción de la salud y la prevención de la enfermedad (Nebot, Rosales, y Borrell, 2009).

\section{Método}

\section{Diseño}

El estudio tuvo enfoque cuantitativo, de tipo experimental y longitudinal, con diseño cuasi experimental, desarrollado en dos grupos, uno experimental y otro de control.

\section{Población y área de estudio}

Este estudio, se desarrolló en el Consejo Regional XII de Huánuco del Colegio de Enfermeros del Perú, durante el año 2017, en la jurisdicción del Departamento de Huánuco. La muestra estuvo conformada por 58 enfermeros para el grupo control y 58 enfermeros para el grupo experimental, quienes cumplieron con los siguientes criterios: enfermeros próximos a colegiarse en el CR XII de Huánuco, próximos a realizar el SERUMS que además aceptaron firmar el consentimiento informado. Se procedió a seleccionar los grupos de intervención (experimental y control) de forma aleatoria.

\section{Recolección de datos}

La encuesta fue la técnica usada para la recolección de datos, mientras que el instrumento usado fue cuestionario de desempeño del rol comunitario que constó de seis dimensiones: promoción de la salud, estimulación temprana, atención inmediata del recién nacido, crecimiento y desarrollo del niño, estrategia sanitaria de inmunización, liderazgo y gestión del cuidado humanitario. Dicho instrumento fue validado por 2 jueces y 5 expertos. Para el cálculo de la confiabilidad se realizó una prueba piloto a 15 enfermeros ajenos a la muestra en estudio, con los cuales se elaboró una base de datos que permitió el análisis de consistencia interna del cuestionario, mediante la prueba de KR de Richardson, donde se determinó el nivel 
de confiabilidad; habiéndose obtenido el valor de 0, 87. El marco muestral se obtuvo de la plantilla de licenciados candidatos a la colegiatura de enfermería en el CR XII de Huánuco.

\section{Desarrollo del programa de la formación continúa}

La intervención se basó en la Norma Técnica de Salud para la Atención Integral de Salud de la Niña y el Niño NTS N 040-MINSA/DGSP.V.01, que establece "Fortalecer las competencias para la atención integral de salud y la implementación del Plan Esencial de Aseguramiento en Salud (PEAS), con enfoque intercultural de los equipos de Salud/RHUS/Personal de Salud/trabajador de salud del Sistema Nacional Coordinado y Descentralizado de Salud, en el marco del proceso de la descentralización, el Aseguramiento Universal y los objetivos estratégicos del Sector" (MINSA, 2008, p. 17).

Posteriormente se dio a conocer el cronograma de las sesiones de fortalecimiento de las competencias en el cuidado comunitario, el cual tuvo una duración de 51 horas, con una periodicidad de 3 veces por semanas durante un mes. Los temas dictados fueron: promoción de la salud, estimulación temprana, atención inmediata del recién nacido, crecimiento y desarrollo del niño, inmunización y liderazgo, y gestión del cuidado humanitario. Para cada curso se contó con expertos, especialistas en el área.

\section{Aspectos éticos}

Por ser un estudio no experimental no se han transgredido los derechos humanos de las personas, ya que, en las encuestas elaboradas e incluidas en el estudio, la identidad permaneció en absoluta reserva. Además, se tuvieron en cuenta los principios bioéticos (beneficiencia, no maleficiencia, justicia y autonomía) y toda la información utilizada para su realización fue tratada con total confidencialidad. Se protegió la privacidad de los participantes en el estudio y se garantizó que los datos obtenidos fueran utilizados exclusivamente con fines investigativos. Previo al reclutamiento para el estudio, se obtuvo el consentimiento informado por escrito de todos los participantes.

\section{Análisis de datos}

Se analizó por medio de los valores numéricos alcanzados en las variables en estudio, para el cual se aplicó la prueba t de Student (prueba paramétrica, donde hubo 
normalidad en la distribución de las variables contrastadas), la cual discriminó si hay relación entre estas dos variables. Del mismo modo, se consideró el p-valor < 0,05 (a $=5 \%)$, para rechazar o aceptar la hipótesis nula. En el procesamiento de los datos se utilizó el paquete estadístico Statiscal Package for the Social Sciences (SPSS), versión 20.0 para Windows.

\section{Resultados}

Respecto a los grupos de edad se identificó que el 25,9\% (15) de los enfermeros serumistas del grupo experimental tenían entre 22 y 24 años, el 34,5\% (20) entre 25 a 27 años, y el 39,7\% (23) entre 28 a 30 años; mientras que en el grupo de control el 34,5 \% (20) tenían entre 22 y 24 años; el 25,9\% (15) entre 25 y 27 años, y el 39,7\% (23) entre 28 a 30 años. En cuanto al género se observó que el 19\% (11) del grupo experimental pertenecían al género masculino, frente al $81 \%$ (47) que lo hacían al femenino; en el grupo control se observó que el 22,4\% (13) pertenecían al género masculino, frente al $77,6 \%$ (45) que lo hacían al femenino. La procedencia universitaria de los enfermeros serumistas, fue un 70,7\% (41) de una universidad pública, frente a un $29,3 \%$ (17) privada; mientras que en el grupo control, un 75,9\% (44) procedían de una universidad pública y un 24,1\% (14) privada.

En el análisis descriptivo del desempeño del rol comunitario, según dimensiones, se observó que anterior a la educación continua, el grupo experimental mostraba un desempaño bueno y, en cierto modo, deficiente; mientras que el grupo control Tenia nivel alto y bueno, llevando ventaja éste último grupo. Las dimensiones analizadas y que tuvieron niveles de desempeño inferiores a grupo control fueron: promoción de la salud, estimulación temprana del niño menor de cinco años, crecimiento y desarrollo del niño e inmunización. Tan solo en una dimensión (liderazgo y gestión del cuidado humanitario, con un $86,2 \%$ ) aventajaron en el nivel bueno al grupo de control. Ambos grupos coincidieron, no obstante, en los porcentajes de evaluación en nivel bueno respecto a las dimensiones: promoción de la salud, con un 44,8\% (26) y estimulación temprana del niño menor de cinco años, con un 81 \% (47) (ver tabla 1). 


\section{Tabla 1}

Desempeño del rol comunitario, según dimensiones, en los grupos experimental y control, antes de la educación continua; Huánuco, 2017

\begin{tabular}{llcccc}
\hline \multirow{2}{*}{$\begin{array}{l}\text { Dimensiones } \\
\text { comunitario }\end{array}$} & rol & Nivel de & \multicolumn{4}{c}{ Grupos de estudio } \\
\cline { 3 - 6 } & desempeño & \multicolumn{2}{c}{ Experimental } & \multicolumn{3}{c}{ Control } \\
\cline { 3 - 6 } Promoción de la salud & Alta & 12 & 20,7 & 26 & 44,8 \\
& Buena & 26 & 44,8 & 26 & 44,8 \\
& Deficiente & 20 & 34,5 & 6 & 10,3 \\
Estimulación temprana & Alta & 9 & 15,5 & 10 & 17,2 \\
del niño menor de cinco & Buena & 47 & 81,0 & 47 & 81,0 \\
años & Deficiente & 2 & 3,4 & 1 & 1,7 \\
\multirow{4}{*}{ Atención inmediata del } & Alta & 3 & 5,2 & 5 & 8,6 \\
recién nacido & Buena & 20 & 34,5 & 19 & 32,8 \\
& Deficiente & 35 & 60,3 & 34 & 58,6 \\
Crecimiento y desarrollo & Alta & 9 & 15,5 & 7 & 12,1 \\
del niño & Buena & 47 & 81,0 & 50 & 86,2 \\
& Deficiente & 2 & 3,4 & 1 & 1,7 \\
Inmunización & Alta & 3 & 5,2 & 5 & 8,6 \\
& Buena & 19 & 32,8 & 22 & 37,9 \\
Liderazgo y gestión del & Alta & 36 & 62,1 & 31 & 53,4 \\
cuidado humanitario & Buena & 7 & 12,1 & 8 & 13,8 \\
& Deficiente & 50 & 86,2 & 47 & 81,0 \\
\hline Total & & 1 & 1,7 & 3 & 5,2 \\
\hline Noficiente & 58 & 100,0 & 58 & 100,0 \\
\hline
\end{tabular}

Nota. Cuestionario del rol comunitario

En la evaluación posterior a la educación continua, en el grupo experimental de enfermeros serumistas, se halló un incremento en el nivel de desempeño del rol comunitario respecto al grupo control. Así, en un $89,7 \%$ de caos los puntajes se ubicaron en el nivel alto, lo que supone que ponderan la atención primaria de la salud con actividades principalmente de promoción de la salud; mientas, un 75,9\% se ubicó en el nivel alto del desempeño en estimulación temprana. Lo mismo sucedió en el desempeño del Programa de Crecimiento y Desarrollo del Niño menor de cinco años y con $89,7 \%$ en el liderazgo y gestión del cuidado humanitario. De otro lado, en el grupo control los desempeños se mantuvieron en el nivel bueno, respecto a las dimensiones de las funciones en atención primaria de la salud; solo en la promoción de la salud un 56,9\% (33) evidenció nivel alto de desempeño (ver tabla 02). 


\section{Tabla 2}

Desempeño del rol comunitario, según dimensiones, en los grupos experimental y control, después de la educación continua; Huánuco, 2017

\begin{tabular}{|c|c|c|c|c|c|}
\hline \multirow{3}{*}{ Dimensiones } & \multirow{3}{*}{$\begin{array}{l}\text { Nivel de } \\
\text { desempeño }\end{array}$} & \multicolumn{4}{|c|}{ Grupos de estudio } \\
\hline & & \multicolumn{2}{|c|}{ Experimental } & \multicolumn{2}{|c|}{ Control } \\
\hline & & $\mathrm{fi}$ & $\%$ & $\mathrm{fi}$ & $\%$ \\
\hline \multirow{3}{*}{ Promoción de la salud } & Alta & 52 & 89,7 & 33 & 56,9 \\
\hline & Buena & 5 & 8,6 & 13 & 22,4 \\
\hline & Deficiente & 1 & 1,7 & 12 & 20,7 \\
\hline \multirow{3}{*}{ Estimulación temprana } & Alta & 44 & 75,9 & 12 & 20,7 \\
\hline & Buena & 14 & 24,1 & 46 & 79,3 \\
\hline & Deficiente & 0 & 0,0 & 0 & 0,0 \\
\hline \multirow{3}{*}{$\begin{array}{l}\text { Atención inmediata del } \\
\text { recién nacido }\end{array}$} & Alta & 13 & 22,4 & 6 & 10,3 \\
\hline & Buena & 45 & 77,6 & 42 & 72,4 \\
\hline & Deficiente & 0 & 0,0 & 10 & 17,2 \\
\hline \multirow{3}{*}{$\begin{array}{l}\text { Crecimiento y desarrollo } \\
\text { del niño }\end{array}$} & Alta & 44 & 75,9 & 7 & 12,1 \\
\hline & Buena & 14 & 24,1 & 51 & 87,9 \\
\hline & Deficiente & 0 & 0,0 & 0 & 0,0 \\
\hline \multirow{3}{*}{ Inmunización } & Alta & 13 & 22,4 & 3 & 5,2 \\
\hline & Buena & 45 & 77,6 & 32 & 55,2 \\
\hline & Deficiente & 0 & 0,0 & 23 & 39,7 \\
\hline \multirow{3}{*}{$\begin{array}{l}\text { Liderazgo y gestión del } \\
\text { cuidado humanitario }\end{array}$} & Alta & 52 & 89,7 & 6 & 10,3 \\
\hline & Buena & 5 & 8,6 & 52 & 89,7 \\
\hline & Deficiente & 1 & 1,7 & 0 & 0,0 \\
\hline Total & & 58 & 100,0 & 58 & 100,0 \\
\hline
\end{tabular}

Nota. Cuestionario del rol comunitario

Al evaluar el desempeño del rol comunitario de los enfermeros serumistas entre grupos y momentos del estudio, se observó en el grupo experimental que; antes de la educación continua, el 1,7 \% (1) tenía un alto nivel de desempeño, el 89,7 \% (52) un buen nivel y el 8,6 \% (5) nivel deficiente; por otro lado, el 98,3\% (57) del grupo control tenía un buen nivel y el 1,7\% (1) un nivel deficiente. Después de la educación continua brindada, el grupo experimental evidenció que el 86,2\% (50) tenía un alto nivel de desempeño y el 13,8\% (8) un buen nivel; mientras que el 1,7\% (1) del grupo control, alcanzó alto nivel y el 98,3\% (57) un buen nivel de desempeño del rol comunitario (ver tabla 3). 


\section{Tabla 3}

Nivel de desempeño del rol comunitario según grupos y momentos del estudio; Huánuco, 2017

\begin{tabular}{lcccc}
\hline \multirow{2}{*}{ Nivel de desempeño del rol comunitario } & \multicolumn{4}{c}{ Grupos de estudio } \\
\cline { 2 - 5 } & \multicolumn{2}{c}{ Experimental } & \multicolumn{2}{c}{ Control } \\
\cline { 2 - 5 } & $\mathrm{fi}$ & $\%$ & $\mathrm{fi}$ & $\%$ \\
\hline Antes & 1 & 1,7 & 0 & 0,0 \\
Alta & 52 & 89,7 & 57 & 98,3 \\
Buena & 5 & 8,6 & 1 & 1,7 \\
Deficiente & & & & \\
Después & 50 & 86,2 & 1 & 1,7 \\
Alta & 8 & 13,8 & 57 & 98,3 \\
Buena & 0 & 0,0 & 0 & 0,0 \\
Deficiente & 58 & 100,0 & 58 & 100,0 \\
\hline Total & & & & \\
\hline
\end{tabular}

Nota. Cuestionario del rol comunitario

Al comparar los puntajes promedios del desempeño del rol comunitario de los enfermeros serumistas del grupo experimental, según momentos del estudio, se obtuvo un error típico de la media de 0.16883 puntos (DE = 1.28576). Al aplicar la prueba $t$ de Student para la comprobación de las diferencias de los valores promedios durante la pre y post intervención, los resultados evidenciaron diferencias significativas $[t=-19,301 ; p=0,000]$, siendo mayor en la post intervención; por lo que se admite que la educación continua brindada, fortalece el desempeño del rol comunitario de los enfermeros serumistas del grupo experimental (ver tabla 4).

\section{Tabla 4}

Prueba t de Student de comparación de medias del desempeño del rol comunitario de los enfermeros serumistas del grupo experimental, según los momentos del estudio; Huánuco, 2017

\begin{tabular}{|c|c|c|c|c|}
\hline \multirow[b]{2}{*}{$\begin{array}{l}\text { Comparación de medias de los } \\
\text { momentos del estudio }\end{array}$} & \multicolumn{4}{|c|}{ Valor de prueba $=58$} \\
\hline & IC 95\% & $\begin{array}{l}\text { Error típico } \\
\text { de la media }\end{array}$ & $\dagger$ & p-valor \\
\hline $\begin{array}{l}\text { Promedio de los puntajes del } \\
\text { desempeño del rol comunitario de los } \\
\text { serumistas del grupo experimental } \\
\text { durante la pre y post educación } \\
\text { continua. }\end{array}$ & $(-3,26--1,29)$ & 1,1688 & $-19,301$ & 0,000 \\
\hline
\end{tabular}

Nota. Cuestionario del rol comunitario 


\section{Figura 1}

Medias del desempeño del rol comunitario de los enfermeros serumistas del grupo experimental, durante los momentos del estudio; Huánuco, 2017

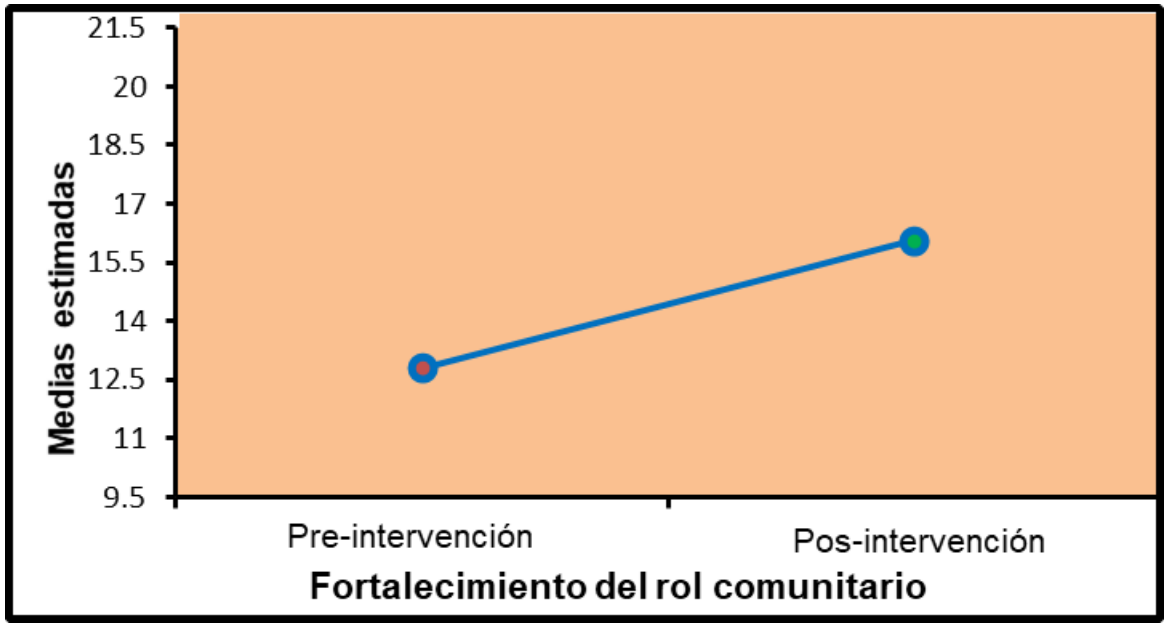

Al comparar los puntajes promedios del desempeño del rol comunitario entre los grupos de estudio y durante la post educación continua, se tuvo un error típico de la media de 0.115454 puntos (DE = 1,17695). Al aplicar la prueba $\boldsymbol{t}$ de Student para la comprobación de las diferencias de promedios, se obtuvo una alta significancia estadística, por lo que se tuvo que rechazar la hipótesis nula de igualdad, admitiéndose por tanto, que los mayores valores promedios logrados por el grupo experimental responden a la educación continua $[t=15,601 ; p=0,000]$ (ver tabla 5).

Tabla 5

Comparación de medias del desempeño del rol comunitario de los enfermeros serumistas según grupos de estudio y durante la pos intervención; Huánuco, 2017

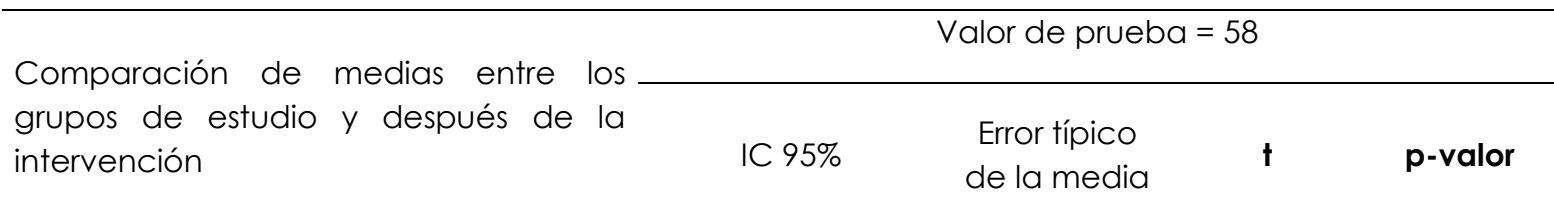

Promedio de fortalecimiento en los

$(2,10-2,72)$

0,1545

15,601

0,000

grupos experimental y control después

de la intervención

Nota. Cuestionario del rol comunitario 


\section{Figura 2}

Comparación de medias del desempeño del rol comunitario de los enfermeros serumistas, según grupos y durante la pos intervención; Huánuco, 2017

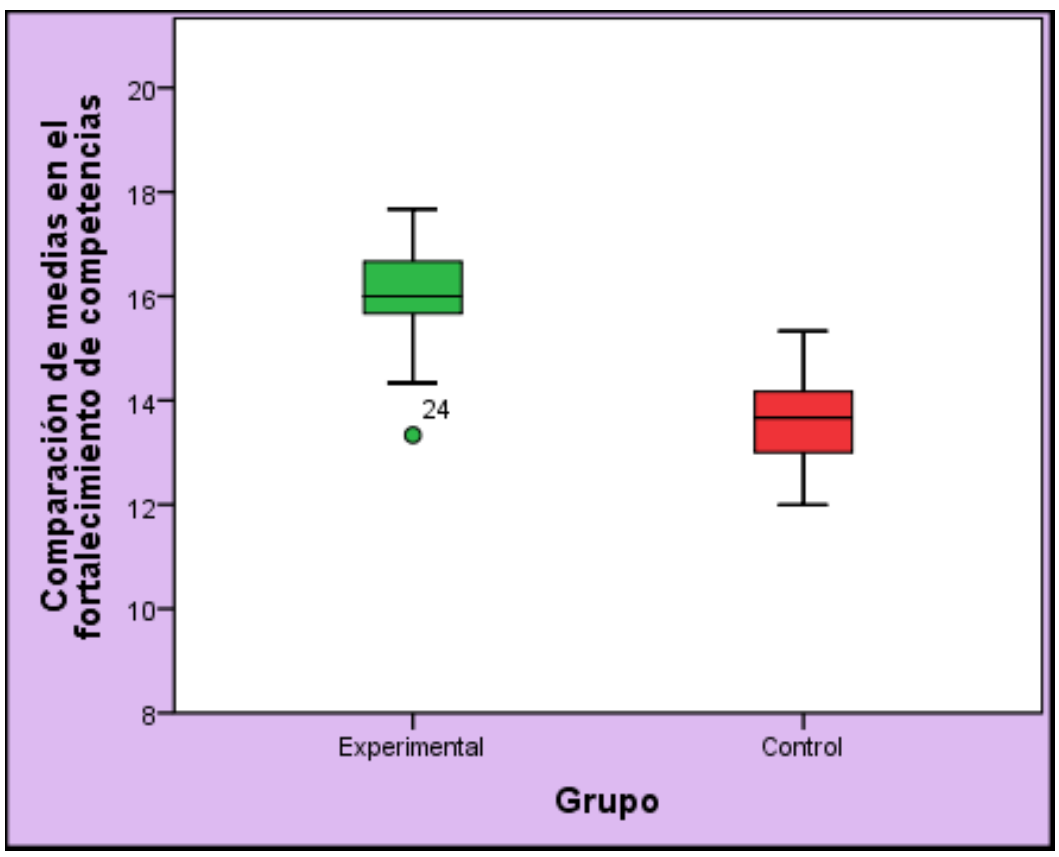

\section{Discusión}

El presente estudio se ubica en el marco del proceso de modernización de la salud, que busca que la atención integral del usuario sea de calidad, a fin de lograr la satisfacción de sus necesidades y expectativas. Para lo cual se requiere que los diversos trabajadores del sector salud, evidencien el mejor desempeño en los diversos roles que les compete (Ministerio de Salud, 2017b).

En el mismo análisis, uno de los objetivos del Ministerio de Salud, (2017a), es alcanzar la excelencia de la atención en los servicios de salud. Lo que se traduce en los esfuerzos por lograr que los usuarios internos y externos perciban la calidad, se incremente la satisfacción de los usuarios externos e internos con el servicio que se brinda; de modo que la formación continua, mediante capacitación con enfoque de resolución de problemas de la realidad, demuestra el desarrollo del personal en las organizaciones. Esto, también fue observado en los programas de formación en Brasil, en el área andina y, particularmente en la Región lca, en Perú, en el año 2008. 
A continuación, se interpretan los resultados, contrastando con estudios similares, contradictorios y las teorías que los sustentan.

Los resultados de la presente investigación ponen en evidencia las diferencias significativas entre los grupos experimental y control, durante los momentos del estudio, en torno al desempeño del rol comunitario de los enfermeros serumistas. Este hecho se explica por el aporte significativo del fortalecimiento de las intervenciones esenciales de enfermería que son orientadas principalmente hacia los niños menores de 5 años en condición de riesgo y vulnerabilidad de la Región Huánuco. Tal resultado indica que la educación continua incrementa en los enfermeros la motivación por un buen desempeño laboral (Böhrt, 2000), cuyos servicios se orientan a la comunidad demandante. Este resultado se corrobora con Nebot Adell et al. (2009), que para que el ejercicio del cuidado comunitario se constituya en eje articulador del sistema de salud en el marco de la APS, es preciso que sus profesionales tengan una sólida formación que les permita responder oportuna y efectivamente a las necesidades de la población. La responsabilidad de su rol comunitario implica capacidad para trabajar en equipo, conocimiento técnico para brindar atención médica apropiada y habilidades para comunicarse con los otros miembros del equipo y con la comunidad. Los profesionales que brindan estos servicios deben contar además, con líderes y tomadores de decisión que conozcan y entiendan los beneficios potenciales de la APS; no sólo en términos de mejora del estado de salud de la población, sino también contribuyendo a un funcionamiento más eficiente del sistema sanitario.

Del mismo modo, los aportes del MINSA, valoran la contribución de la capacitación integral brindada al potencial humano en salud, desde un enfoque de resolución de problemas de la realidad, partiendo de su identificación, análisis y reflexión, seguidos de propuestas de solución, a fin de asegurar su implementación (Ministerio de Salud, 2016, p. 49-50).

Uno de los estudios que coincide con el presente hallazgo, es el realizado por Silva (2018, p, 84), quien observó que los participantes del curso de educación continua adquirieron habilidades y competencias en relación a la actualización de vacunas para el equipo de enfermería y los principales conocimientos (teórico-prácticos) 
adquiridos fueron el esquema de vacunación (32,1 \%), los efectos adversos (21,4 \%) y la organización de una sala de vacunación ideal (14,3\%).

También los hallazgos de Puntunet y Domínguez (2008) ponen en evidencia la capacidad de la educación continua en cubrir las necesidades académicoformativas, de manera pertinente y oportuna, en los profesionales de enfermería; ya que además de ser una estrategia de fortalecimiento, el personal que cuenta con capacitación continua, valoriza su trabajo, es proactivo, analítico, mejora la productividad y, lo más importante de todo, proporciona cuidados seguros y de calidad que se reflejan en mejores resultados en los pacientes. Resulta evidente y de vital importancia la implementación del pensamiento crítico y analítico en el proceso educativo, no sólo del profesional de enfermería, sino de cualquier profesión que desee mantenerse a la vanguardia.

Por su parte, la Federación de Asociaciones de Enfermería Comunitaria y Atención Primaria (2001), explica que la eficiencia en el cuidado del paciente depende de la formación académica, las experiencias vividas y la capacidad física y mental que tenga el profesional de enfermería; al mismo tiempo que este debe cubrir sus propias necesidades: reconocimiento laboral, como estímulo necesario; estado físico y de ánimo; cuidados, salud, comodidad y autoestima para poder rendir y ejercer correctamente su trabajo. Esta es una profesión que amerita crédito a partir de la identidad y el reconocimiento del personal, así como su diferenciación hacia los otros.

Finalmente, se reconoce que el proceso de inducción a través de la formación continua es de suma importancia para los enfermeros recién egresados de las universidades, ya que este permite que este adecuadamente capacitado para el ejercicio de sus funciones en el marco de la APS, manteniendo en todo tiempo el más alto estándar de cuidado de enfermería y de la conducta profesional. Tal como indican Julón y León, (2017), un profesional capacitado e inducido conlleva a una serie de beneficios en diversos servicios, tanto asistenciales como administrativos, ya que la enfermería tiene conciencia de su responsabilidad ante la calidad del cuidado que presta al paciente, a la institución, a la ética, a las leyes y a las normas de la profesión. La contribución del estudio es el beneficio directo al novel profesional de enfermería que postula al SERUMS, pues le ayudará a lograr una mayor 
44

capacidad de adaptación e introducción al nuevo puesto, mejorando así a los resultados sanitarios de los establecimientos de salud del MINSA y EsSalud; fortaleciendo por ende la competitividad y visibilidad del ejercicio profesional de la enfermería.

\section{Agradecimientos}

Este estudio ha sido posible gracias al apoyo del Consejo Regional XII de Huánuco del Colegio de Enfermeros del Perú. Asimismo, se expresa agradecimiento a los enfermeros que aceptaron participar del estudio.

\section{Referencias}

Alarcón, A., Miranda, G. y Palacios, N. (2005). Diagnóstico comunitario en Salud. Asentamiento Lagos de Occidente, 2(1), 123-175.

Böhrt, M. R. (2000). Capacitación y desarrollo de los recursos humanos: reflexiones integradoras. Revista Ciencia y Cultura, 1 (8), 123-131.

Castillon, Y. B. (2015). Desempeño del profesional de enfermería en la atención del puerperio durante el servicio rural y urbano marginal de salud, en Establecimientos de Salud I-1 de las Redess Melgar, Azángaro y Puno 2014 (tesis de pregrado). Universidad Nacional del Altiplano Puno. Facultad de Enfermería. Escuela Profesional de Enfermería, Puno.

Dirección General de Gestión del Desarrollo de Recursos Humanos del MINSA (2017). Competencias Laborales para la mejora del desempeño de los Recursos Humanos en Salud. Recuperado de http://bvs.minsa.gob.pe/local/minsa/1877.pdf

Federación de Asociaciones de Enfermería Comunitaria y Atención Primaria (2001). Justificación de la necesidad de desarrollo de la Especialidad de Enfermería de Salud Comunitaria. Recuperado de http://www.indexf.com/lascasas/documentos/lc0060.php

Feliu, E. (1998). Leyes nacionales e internacionales y su relación con el personal de enfermería. La Habana, 1 (1), 23-45. 
Gómez, E. M. y Llontop, R. M. (2014). Cuidado brindado por la enfermera serums durante el puerperio mediato, puestos de salud: Olmos y Salas 2013 (tesis de pregrado).

Julón, L. y León, I. E. (2017). Diseño e implementación de un programa de inducción para mejorar el nivel de conocimientos y satisfacción del nuevo profesional de enfermería del Hospital José Agurto Tello. Lurigancho-Lima, 2015 (tesis de pregrado).

Ministerio de Salud (1999). Programa de Fortalecimiento de Servicios de Salud.

"Cursos de Gestión en redes de establecimientos y servicios de salud".

Recuperado de

http://www.bvsde.paho.org/bvsars/e/fulltext/tecno/tecno.pdf

Ministerio de Salud (2017a). Gestión de la capacitación en la organización.

Recuperado de

http://www.minsa.gob.pe/publicaciones/pdf/capacitacion.pdf

Ministerio de Salud (2017b). Módulo de Capacitación en Nutrición: Orientación y manejo alimentario nutricional. Recuperado de http://bvs.minsa.gob.pe/local/PSNB/69_modcapac.pdf Ministerio de Salud (2016). Programa Nacional de Formación en Salud Familiar y Comunitaria. 2da. ed., 2016. 208 p. P 49-50. Perú. Recuperado de https://www.aics.gov.it/wp-content/uploads/2018/07/PERU_profam_2016.pdf Ministerio de Salud (2008). Norma Técnica de Salud "Atención integral de Salud de la Niña y el Niño". Lima: Ministerio de Salud. Dirección General de Salud de las Personas. Dirección de Atención Integral de Salud.

Ministerio de Salud Pública (2008). Capacitación y desarrollo de los recursos humanos. Recuperado de http://www.sld.cu/galerias/pdf/sitios/cpich/manual_de_capacitacion._tomo_ii.pdf

Nebot, C., Rosales, C. y Borrell, R. M. (2009). Desarrollo de competencias en atención primaria de salud. Rev Panam Salud Publica, 26(2), 176-183.

Organización Panamericana de la Salud (2008). Sistemas de salud basados en la Atención Primaria de Salud. Estrategias para el desarrollo de los equipos de APS. Washington, D.C.: Organización Panamericana de la Salud. 
Ortiz-Aguí, M., Palacios-Flores, E., y Garay Cabrera, G. R. (2019, marzo 2). La Estrategia de educación continua en la calidad del desempeño de los enfermeros del Perú. Revista Peruana De Ciencias De La Salud, 1 (1), 36-44. https://doi.org/https://doi.org/10.37711/rpcs.2019.1.1.5

Puntunet, M; Domínguez, A. (2008). La educación continua y la capacitación del profesional de enfermería. Revista Mexicana de Enfermería Cardiológica, 16 (3): 115-117. Recuperado de https://www.medigraphic.com/pdfs/enfe/en2008/en083g.pdf

Ramos, R., y Robles, B. (2016). Vivencias percibidas del fortalecimiento en valores para el cuidado humano de los estudiantes de la Facultad de Enfermería de la UNHEVAL 2015 (tesis de pregrado). Universidad Nacional Hermilio Valdizán. Facultad de Enfermería. Escuela Académico Profesional de Enfermería, Huánuco.

Saavedra, M. E. (2014). Participación social en la prevención y promoción de la salud y en el proceso de municipalización desde la perspectiva de la enfermera. Región Lambayeque (tesis de doctorado). Universidad Nacional de Trujillo. Escuela de Postgrado. Programa Doctoral en Ciencias de Enfermería, Trujillo.

Silva, E. (2018). Educación continua para el equipo de enfermería sobre Calendario Nacional de Vacunación, Municipio de Santa María do Pará. Revista Internacional de Apoyo a la Inclusión, Logopedia, Sociedad y Multiculturalidad, 4(2), 70-84.

Torres, M., Dandicourt, C. y Rodríguez, A. (2005). Funciones de enfermería en la atención primaria de salud. Revista Cubana de Medicina General Integral, $21(3-4)$.

Willamowius, D. y Misue, L. (2009). Validação de conteúdo de indicadores de qualidade para avaliação do cuidado de enfermagem. Revista da Escola de Enfermagem da USP, 43(2), 34-56.

Zarate, R. A. (2004). La gestión del cuidado de enfermería. Index de enfermería, 13(2), $42-46$. 